\title{
Referencia oportuna para la atención de SARS-CoV-2. La hipoxemia silenciosa del COVID-19
}

\section{Timely reference for the attention of SARS-CoV-2. The management of silent hypoxemia of COVID-19}

\author{
Oscar Emmanuel Grimaldo, ${ }^{*}$ Solange Deianira Azpitarte ${ }^{\ddagger}$
}

\begin{abstract}
*Universidad de Celaya, Rancho Pinto, Celaya, México; ${ }^{\ddagger}$ Unidad Operativa de Hospitalización del $8^{\circ}$ Régimen Blindado, $2^{\circ}$ Nivel de Atención Médica, SEDENA, Irapuato, México.
\end{abstract}

Vivimos una situación inédita en la historia de la medicina, la cual está siendo producida por la pandemia de COVID-19 y que ha modificado la situación de los sistemas de salud en el ámbito mundial. Esta situación también tendrá una continuación, modificando la forma en la que nos enfrentaremos a las enfermedades respiratorias y al modo particular en que valoraremos a los pacientes con estas patologías. ${ }^{1}$

Nuestro país presenta otras grandes dificultades, como la enorme cantidad de personas susceptibles a la enfermedad y la escasa destinación de recursos a su atención por parte del sector salud. La detección oportuna es limitada, ya que los signos y síntomas son parecidos a los de otras enfermedades de vías aéreas respiratorias superiores. Por ello, la reciente enfermedad COVID-19 ha traído consigo un verdadero reto, tanto en salud como en los aspectos básicos de la vida cotidiana y, sin duda, se ha vuelto una lucha para el sector médico. Una de las interrogantes médicas más relevantes que podrían ayudar a identificar a pacientes susceptibles de la enfermedad es la denominada hipoxemia silenciosa.

Los pacientes con enfermedad por coronavirus (COVID-19) presentan niveles de oxígeno incompatibles con la vida sin presentar disnea. Este hecho es especialmente desconcertante para los médicos, y se considera que desafía la biología básica. Esta situación ha atraído una amplia cobertura en

Correspondencia:

Dr. Oscar Emmanuel Grimaldo

Universidad de Celaya, Rancho Pinto, Celaya, México.

Correo electrónico: 11630@udec.edu.mx

Trabajo recibido: 30-VIII-2020; aceptado: 11-IX-2020.

Citar como: Grimaldo OE, Azpitarte SD. Referencia oportuna para la atención de SARS-CoV-2. La hipoxemia silenciosa del COVID-19. Neumol Cir Torax. 2021; 80 (1): 70-71. https://dx.doi.org/10.35366/99459 los medios, pero no se ha discutido en revistas médicas. Es posible que el coronavirus tenga una acción sobre los receptores implicados en la quimiosensibilidad al oxígeno. Sin embargo, el establecimiento de los mecanismos fisiopatológicos puede explicar la mayoría, si no todos, los casos de hipoxemia silenciosa. ${ }^{2}$

Estos mecanismos incluyen: a) la forma en que la disnea y los centros respiratorios responden a niveles bajos de oxígeno; b) la forma en que la tensión de dióxido de carbono predominante $\left(\mathrm{PaCO}_{2}\right)$ atenúa la respuesta del cerebro a la hipoxia; c) efectos de la enfermedad y la edad en el control de la respiración; d) inexactitud de la oximetría de pulso a bajas saturaciones de oxígeno; y e) cambios inducidos por la temperatura en la curva de disociación del oxígeno. ${ }^{2,3}$

Una vez entendido el concepto de la hipoxemia silenciosa, es posible obtener beneficios en la detección oportuna de la enfermedad si en un primer nivel se presta atención a la saturación de $\mathrm{O}_{2}$ con un oxímetro, además de la evaluación de los signos y síntomas ya establecidos por la Organización Mundial de la Salud. En México, por sus variables sociodemográficas, un alto número de habitantes radica en zonas rurales en donde se encuentran situados a una altura considerable sobre el nivel del mar, donde además tienen condiciones socioeconómicas desfavorables y están en exposición al humo de la leña, ${ }^{4,5}$ es aquí, en el primer nivel de atención, donde es atendido un alto porcentaje de pacientes potenciales. De esta manera, se podría dar seguimiento y, en su momento, referir de manera oportuna a estos casos y no esperar a que acontezcan los desenlaces de la enfermedad.

En conclusión, las características de COVID-19 que los médicos encuentran desconcertantes se vuelven menos extrañas cuando se ven a la luz de los principios bien establecidos en la fisiología respiratoria. Una mejor comprensión de estos mecanismos hará más eficiente la atención al 
paciente y tal vez conducirá a la derivación oportuna del paciente para la atención de la enfermedad con el uso de oximetría de pulso, además de la exploración física.

\section{REFERENCIAS}

1. Tobin MJ, Laghi F, Jubran A. Why COVID-19 silent hypoxemia is baffling to physicians. Am J Respir Crit Care Med. 2020;202(3):356360. doi: 10.1164/rccm.202006-2157CP.

2. Jounieaux V, Parreira VF, Aubert G, Dury M, Delguste P, Rodenstein DO. Effects of hypocapnic hyperventilation on the response to hypoxia in normal subjects receiving intermittent positivepressure ventilation. Chest. 2002;121(4):1141-1148. doi: 10.1378/ chest.121.4.1141.
3. Couzin-Frankel J. The mystery of the pandemic's 'happy hypoxia'. Science. 2020;368(6490):455-456. doi: 10.1126/science.368.6490.455

4. Pérez-Padilla JR, Thirión-Romero II, Aguirre-Pérez T, RodríguezLlamazares S. Qué tan silenciosa es la hipoxemia en COVID-19? Neumol Cir Torax. 2020;79(2):69-70. doi:10.35366/94629.

5. Masera O, Riojas-Rodríguez H, Pérez-Padilla R, Serrano-Medrano M, Schilmann A, Ruiz-García V, et al. Vulnerabilidad a COVID-19 en poblaciones rurales y periurbanas por el uso doméstico de leña. 2020. Disponible en: https://www.insp.mx/avisos/5386-vulnerabilidad-covid19-poblaciones-rurales.html

Conflicto de intereses: Los autores declaran no tener conflicto de intereses. 